\title{
Internet of Things-Based Smart Electricity Monitoring and Control System Using Usage Data
}

\author{
Mohammad Kamrul Hasan $\mathbb{D}^{1},{ }^{1}$ Musse Mohamud Ahmed, ${ }^{2}$ Bishwajeet Pandey $\mathbb{D}^{3}{ }^{3}$ \\ Hardik Gohel, ${ }^{4}$ Shayla Islam $\mathbb{D}^{5},{ }^{5}$ and Izzul Fitrie Khalid ${ }^{2}$ \\ ${ }^{1}$ Network and Communication Lab, Center for Cyber Security, Faculty of Information Science and Technology, \\ Universiti Kebangsaan Malaysia (UKM), 43600 UKM, Bangi, Selangor, Malaysia \\ ${ }^{2}$ Department of Electrical and Electronics Engineering, Universiti Malaysia Sarawak, 94300 Kota Samarahan, Sarawak, Malaysia \\ ${ }^{3}$ Department of Computer Science and Engineering, Gyancity Research Ltd., India \\ ${ }^{4}$ Department of Computer Science, University of Houston-Victoria, 3007 N. Ben Wilson, St. Victoria, TX 77901, USA \\ ${ }^{5}$ Institute of Computer Science and Digital Innovation, UCSI University, 56000 Kuala Lumpur, Malaysia
}

Correspondence should be addressed to Mohammad Kamrul Hasan; hasankamrul@ieee.org and Shayla Islam; shayla@ucsiuniversity.edu.my

Received 5 June 2021; Accepted 14 September 2021; Published 27 October 2021

Academic Editor: Deepak Gupta

Copyright (c) 2021 Mohammad Kamrul Hasan et al. This is an open access article distributed under the Creative Commons Attribution License, which permits unrestricted use, distribution, and reproduction in any medium, provided the original work is properly cited.

In everyday life, electricity is necessary, and proper use is critical. To strengthen home electricity control, the existing systems have been examined over the years. However, the existing PMAS method's error ratio is higher and does not allow for a remote monitoring system. Therefore, this study proposes a smart monitoring and control system (SMACS) for household appliances. The application's significance is to monitor household appliances' electricity usage using hardware and the Internet of Things (IoT) methods. The prototype of the proposed system is designed and developed considering Arduino UNO, a liquid crystal display (LCD), an ACS712 current sensor module, relays, and AC sources. The components are selected from the software library, and the simulation results are found the same as the prototype. WiFi module ESP8266 is not included in the design because it is not provided in the system. The data is recorded in cloud storage using Thing-speak. A mobile application (Virtuino) also accesses the data to visualize it through the graphical and numerical display. This study provides users with an easy system to monitor and control household appliances' power consumption using mobile applications. Results show that the proposed system provides $0.6 \%$ current errors for the hairdryer appliance, whereas the existing Power Monitoring and Switching (PMAS) system provides $7.8 \%$ current errors.

\section{Introduction}

In Malaysia, electricity has the highest demand as it is expected to increase gradually in years to come, in line with the expansion of urbanization, rapid industrialization, and the growing population of the country. Statistically, residential sectors consume up to $48 \%$ of the energy globally [1]. Around $40 \%$ of Malaysia's buildings' energy is divided into commercial buildings and residential buildings [1]. In this modern era, people utilize household appliances with new technologies. In Malaysia, on average, 20 to 30 electrical household appliances are used in homes [2]. A vast majority of household appliances consume a large amount of power and energy. Consumers mostly tend to leave their lights, fans, freezer, air conditioner, and other appliances turned on when they are not in use, resulting in energy wastage, a tendency of human behaviour [3].

This negligence concerning the consumers' behaviour can lead to excessive power consumption and wastage of the electrical energy needed, and it can shorten the life span of household appliances such as hair dryer, dry iron, induction and rice cooker, water heater, microwave oven, air 
conditioner, and television. It has been identified that old appliances contribute to higher energy consumption than newer ones.

This paper presents an integration of both hardware and software. The software is used to monitor power usage and the consumption of household appliances and control systems through overcurrent relay and notification of any mismatches. The developed system consists of Arduino UNO, a WiFi module (ESP8266), a relay, a low current sensor breakout (ACS712), and a liquid crystal display (LCD). Arduino UNO is a microcontroller used to program customized coding for executing output at any instant time. It is also a very capable microcontroller that receives and sends information over the Internet with various modules and shield platforms. However, in this case, the ESP8266 WiFi module is used as the platform. The ESP8266 WiFi module is famous for its IoT applications. The relay function performs the cut-off current and the isolation of input and output operations and performs switching functions. The outputs are shown in 2 ways: LCD and IoT implementation based on the web server or mobile application (APPS). LCD is used to display the voltage, current, and power consumption where the web or mobile application is used to visualize the data and trigger alarm, when necessary, In the web and mobile application systems, the energy usage statistics of power consumption parameters are determined. It displays the detailed monitoring of electrical quantities such as voltage, current, power, and energy.

IoT attaches the internet connectivity and remote control of mobile devices, integrated with a range of sensors, to the smart home appliances. Sensors can be embedded in the refrigerator, air conditioner, and lighting, and other environmental sensors can be connected to home-related gadgets. The overcurrent or circuit overloading can be detected earlier based on advanced IoT applications where consumers' alarm triggers.

The significance of SMACS is that it creates an opportunity for consumers to control their power consumption practices and help them manage their power and energy usage. It also creates an opportunity for the consumers to practice energy saving and to keep track of their household appliance's performances and current behaviour to prevent overcurrent. Therefore, the main challenge will be designing an efficient technique that can monitor power consumption in residential buildings.

\section{Literature Review}

A brief overview of the related techniques and literature on the study's scope is discussed in this section. Four (4) components are discussed, such as a monitoring system, a control system, IoT implementation, and other related techniques.

Nowadays, people's dependency on electricity is extreme, as power consumption has increased for the past few years [4]. It is imperative to consider monitoring and measuring the electric system or appliances that operate every day for residential and commercial buildings. As the focus of this article, the residential sectors are made up of small energy consumers and users, such as houses and apartments. The research suggested that the residential energy consumers [5] waste $41 \%$ of the power supplied to their homes. Various research and trials have been shown that on average, savings of $7.8 \%$ to $16.7 \%$ home energy can be achieved by using a home energy monitoring system [6, 7]. Several case studies have been shown that energy consumption can be reduced by modifying the lifestyle with proper habits/behaviours [8-10].

A node microcontroller unit (NodeMCU) with a WiFibased gateway has been used to connect different sensors and update their data to the Adafruit IO cloud server [11]. A power monitoring system can help users or consumers monitor their usage of electricity efficiently. Improvement of electricity efficiency usage can be made by reduction due to changing habits [12]. In this modern era, monitoring systems must sort the data, record data in the system, exploit them to get the intrinsic information shown in legit and innovative ways, and access the Internet to efficiently visualize it [13]. A power monitoring system can be considered a user-friendly and unified solution for reliable electricity management [14]. This system can help users to change their use by providing real-time feedback [15].

The control system is a system that can control other things depending on what needs to be controlled. Local and remote control are included in the control application. Local control is an action that the control unit will take on its own, and remote control is a mechanism that remotely controls the IoT-based system [15]. Electrical equipment operational status monitoring will reduce the cost of building power consumption and increase buildings' electrical energy to a reasonable and efficient ratio [16]. As all can be managed, control systems can help consumers handle their energy more effectively and efficiently. The control system controls the current in this study, which will be cut off if an overcurrent is found. This control system can raise awareness of the consumption of household appliances among users.

Technology is one of the main reasons for a country to become a well-developed country. Innovations and technology can likely change people's lives and make the world more sustainable and inclusive. Internet of Things, known as IoT, is a new and ever-growing network that is becoming a hot topic in people's conversations in the era of modern technology. IoT is a wide-open and complete network of smart and intelligent objects that has the full capability and capacity to autoorganize; share information, data, and resources; and act and react to any circumstances or atmosphere of the changing environment [17-22]. IoT is growing and needs to be sustained so that it will be the expanded, innovative concept in the IT world. IoT advancement controls the real Thing that will transform the Internet into a fully integrated Future Internet [23]. Future information communication technology is to be used with embedded sensors. The target applications are smart metering, Internetconnected sensor devices for household appliances, emphasizing the benefits of remote real-time monitoring for household energy consumption appliances [13, 24-30]. The IoT applications have already brought attention to and shown the 
performance of monitoring modern household appliances' energy usage toward a sustainable and improved quality of urban lifestyle. IoT is beneficial and recommended in smart home applications [31-33]. It has improvised our quality of life and also can reduce the unnecessary costs of daily life. This wellness system has been implemented for many great things and monitoring and controlling the electricity at home. The information is uploaded to the website by a server using the cloud server's local home gateway. However, security and privacy challenges have been raised due to the reliability and dependability issues of the Internet. This is because of some connections between the cyber environment and physical environment to fulfill the consumers' demands. It is presumed that these problems will indeed be solved in the future [34], as shown in Figure 1.

Even though IoT is an intelligent technology that makes people's lives more comfortable, IoT-connected systems might lack a comprehensive platform if applications of individual items are not transformed. These separate individual applications are combined into a single infrastructure, where a platform can be used in the future for data mining and extraction of knowledge [32]. As far as IoT is concerned, the future of IoT will be the best and advanced in the sensing services; communications; and in monitoring, controlling, and producing information from vast amounts of data [8]. Gartner, a technology consulting company, is concerned in predicting the future of technology trends, especially the Internet of Things for smart homes, smart industries, agriculture, healthcare, and autonomous vehicle applications [35-40].

The energy that is mainly being used and consumed in the residential areas is primarily on water heating, lighting, air conditioner, refrigerator, and more. Precisely, in Malaysia, it can be predicted that only a small amount of energy is consumed during office hours between 0800 hours and 1700 hours. However, peak demand reaches mainly at night, where all the people gather around having dinner, watching television, and more. Yet, their power rating can be categorized as household appliances, where Table 1 shows the average household item power rating [39-42].

Moreover, household appliances can also be divided into three (3) parts: inductive, capacitive, and resistive. Resistive load-based heating appliances have the highest energy consumption as compared to inductive and capacitive household appliances. Air conditioners and washing machines use inductive and capacitive loads that can be considered reactive loads. They have lower consumption than the resistive-based loads, but the resistive load's power factor is one. The load needs more current to satisfy the real power requirement if the power factor is low, and most consumers are not aware of it as the electricity bills are basically in energy $(\mathrm{kWh})$ [43].

2.1. Related Works and the Research Challenges. In this section, related IoT-based electricity monitoring systems are discussed and explained. The development of these projects has led the world to a better future in saving electricity, practicing energy saving, and making electricity usage very effective and efficient.

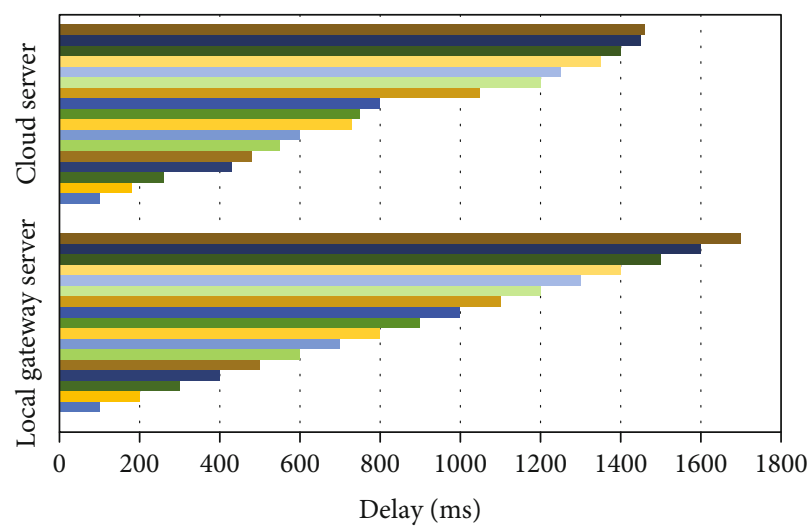

FIgURe 1: Final information uploading delay for cloud and local home gateway approach [34].

Smart Power Monitoring and Analysis is aimed at developing a solution to keep track of every electrical appliance and monitor the energy used consumed by an Android device. As mentioned, this study's main problem statement is that most of the power meters installed in any residential buildings showed the total consumption of the electricity used. It does not mention which appliances contributed to the usage of most electricity. The goal is at least to lower the electricity bills and energy consumption by $30 \%$ to $40 \%$. This can be achieved by a proper monitoring system that allows item-wise energy consumption monitoring and can regulate lifestyle with usage habits [9]. The architecture used only current sensors to measure all the parameters needed and a microcontroller as the system. The data collected and monitored were stored in a central cloud server instantly for some analysis. The IoCare IoT module has been used on the WiFi board with a microcontroller [44]. Authors have presented a system with mobile apps to collect energy consumption data. This study's performance was satisfied as the electricity consumption is successfully shown in their smartphone apps in meters and numeric form; however, a real-time analytical value of the energy or the power consumed has not existed in the system. This application can help a single user forecast energy use and billing with reliable accuracy comprehensively, manage the unnecessary waste of energy consumption and optimize electricity use from a mass-user perspective [44].

As mentioned in this paper, the primary source of the development and advancement of technology in this world is electric energy. There is a demand for it from both domestic and industrial sectors. As stated in the annual energy report, the demand for electricity in homes is expected to increase by $24 \%$ [30]. As in recent years, wireless sensor networks (WSNs) have experienced rapid growth. WSNs are incredibly flexible and provide an exciting opportunity to advance the future of home automation. An IoT-based intelligent energy management system using WSN is a project implemented as IoT for a home appliance monitoring system and real-time power management system. This system integrates WSN and Web Service communications to comprehend the management of a power and information 
TABLE 1: Average household item power rating [39-42].

\begin{tabular}{|c|c|c|}
\hline No & Item & Rating (Watt) \\
\hline 1 & Television-Samsung & 150.00 \\
\hline 2 & Television-Sony & 125.00 \\
\hline 3 & Air conditioner-Panasonic & 600.00 \\
\hline 4 & Air conditioner-Panasonic 2 & 500.00 \\
\hline 5 & Air conditioner-Daikin & 800.00 \\
\hline 6 & WiFi modem & 10.00 \\
\hline 7 & Cable TV setup box & 25.00 \\
\hline 8 & Internet modem & 10.00 \\
\hline 9 & Refrigerator & 150.00 \\
\hline 10 & Water heater & $1,000.00$ \\
\hline 11 & Microwave & 600.00 \\
\hline 12 & Dispenser & 600.00 \\
\hline 13 & Dishwasher & $1,200.00$ \\
\hline 14 & LED light bulbs -7 & 7.00 \\
\hline 15 & LED light bulbs-9 & 9.00 \\
\hline 16 & Incandescent light bulbs -60 & 60.00 \\
\hline 17 & Incandescent light bulbs -80 & 80.00 \\
\hline 18 & Washing machine & 500.00 \\
\hline 19 & Vacuum cleaner & 200.00 \\
\hline 20 & Electric iron & 400.00 \\
\hline 21 & Rice cooker & 400.00 \\
\hline 22 & Toaster & 600.00 \\
\hline 23 & Laptop & 50.00 \\
\hline 24 & Printer & 20.00 \\
\hline 25 & Scanner & 10.00 \\
\hline 26 & Desktop computer-Apple & 100.00 \\
\hline 27 & Mobile phone charger-Samsung & 3.00 \\
\hline 28 & Mobile phone charger-iPhone & 3.00 \\
\hline 29 & Water pump & 400.00 \\
\hline 30 & Electric saver & 15.00 \\
\hline 31 & Lawn mower & $1,000.00$ \\
\hline 32 & Food blender & 300.00 \\
\hline 33 & Hair dryer & 400.00 \\
\hline 34 & Coffee maker & 600.00 \\
\hline
\end{tabular}

provider using the IoT platform. The proposed system for this project is to use the current sensor and voltage sensors for calculation purposes. Figures 2 and 3 refer to the functional description of the system developed. Data is transferred wirelessly with ZigBee (ZigBee Ethernet shield receivers via serial port). The data collected from the Ethernet shield is sent using the WiFi router to the LAN. The system is controlled and monitored remotely [45].

The aim of this research is consistent with SMACS, but this method uses ZigBee and Ethernet.

Ethernet is able to connect to an Ethernet network as either a client or a server [37]. The proposed project uses WiFi module ESP8266, which is a transreceiver module compared to the ZigBee module.

Electrical engineers have to conserve energy in this world as everyone demands electrical energy at any point in the human life cycle. The proposed system designs an autonomous system capable of controlling electricity flow, reducing consumption, and collecting and transmitting maximum and dynamic loads. The system consists of five parts: tracking, calculating, monitoring, regulating, and electrical safety of the power system.

Power system monitoring is a robust, reliable, and highly accurate energy management system, while metering is the important system in which data and information are acquired and presented in the database in real time. The measurements indicate the voltage, current, frequency, active power, reactive power, and apparent power. Monitoring and control is the management of information, and the application of a control system involves the maintenance of information.

Electrical protection is also included in this system to protect the equipment and the personnel and avoid extensive damage caused by faults as it can monitor a large-scale system that is a substation. The proposed system for this project is as follows:

(1) Voltage sensing by using a voltage transformer

(2) Measure current via current sensors

(3) Zero crossing technique used for sensing frequency

(4) Voltage and current signal are used for power factor estimation

(5) The load will be tripped when the current event exceeds the set value

(6) A generator will be switched on and the load will be shifted when there is a shutdown

There are two parts of this study that are crucial for references: the voltage divider circuit and the current divider circuit. The voltage divider circuit is used to scale down the waveform, and an offset is added when there is no harmful component. Single-phase voltage is monitored and calculated by stepping down to $12 \mathrm{~V}$ of $240 \mathrm{~V}$ [14]. On the other hand, the current is measured using the current transformer sensor where the current transformer sensor with burden resistors and biasing voltage dividers (R1 and R2) is used to achieve the wave of the current divider [14]. Therefore, the system design uses the Arduino microcontroller, where a single-phase voltage and current are measured. The corresponding frequency $(50 \mathrm{~Hz})$, power factor, apparent power (KVA), reactive power (KVAR), and real power (KW) are found by calculating the value shown in the LCD [14].

This proposal is close to SMACS as the system for both similar. The main difference between this work [16] and SMACS is that the existing system focused on a bigger scale of the electrical system, a substation, whereas SMACS is narrowed down to the minor part in household electrical appliances. The significance of a microcontroller-based power monitoring and switching device for appliances over a ZigBee Network is the development of a system that can help users be more aware of their appliances' consumption. Another focus of PMAS is storing data and monitoring 


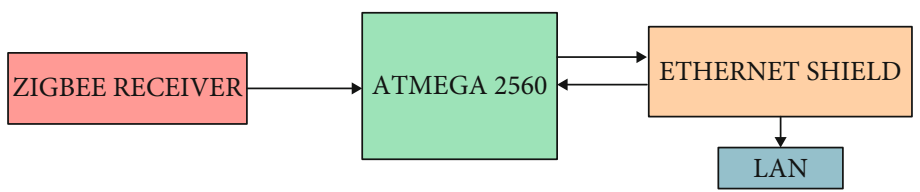

Figure 2: Receiver section of smart power management system [32].

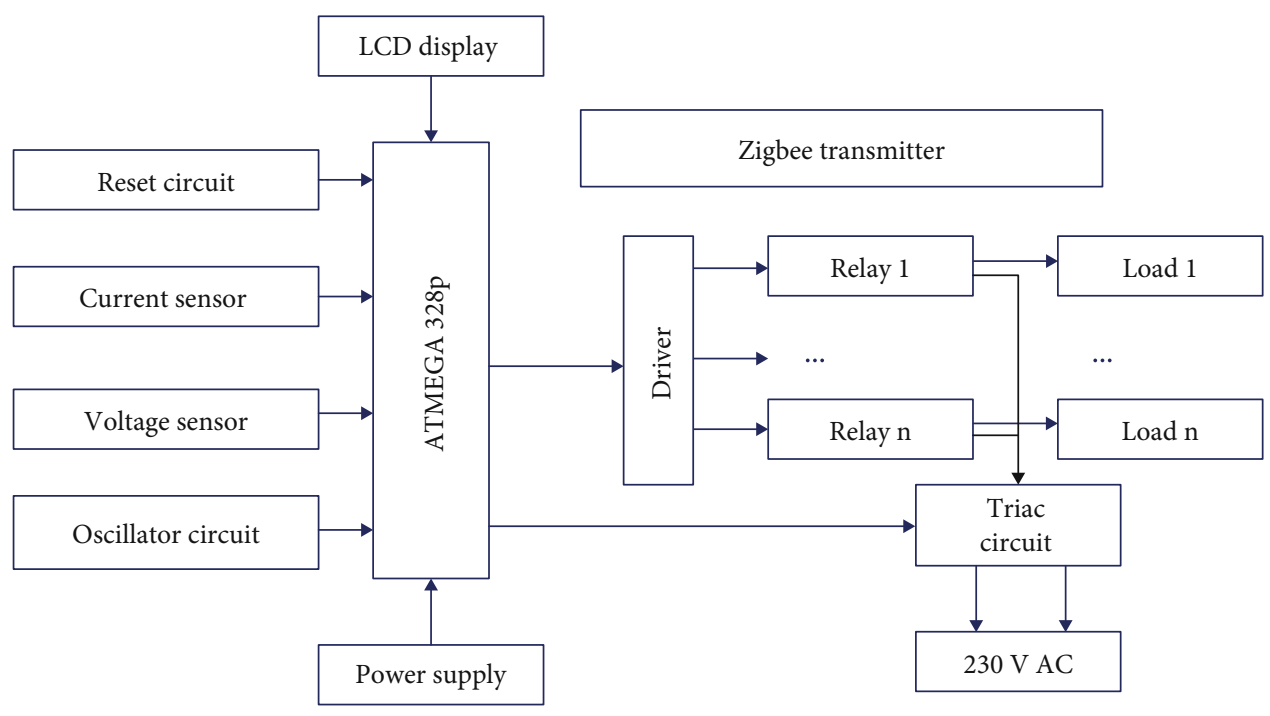

Figure 3: Transmitter section of smart power management system [45].

consumption based on the android app. The microcontroller will read the current and transverse parameters on the android app: voltage, current, and power. Zigbee is used to transmit data but in a small range of frequencies. The gateway sends and receives data to the web server and mobile application [46]. The design is partly divided into adapters, central hubs, databases, and android applications. The adapter consists of the current sensor ACS712, the same current module used in the proposed system. The data is shown in Table 2, where the data collected are based on four appliances.

Table 2 shows the reading from the multimeter compared to the reading with the PMAS converter. It was not far from accurate, but there were some errors in the system. Developing Arduino-based IoT measurement system for energy on-demand monitoring is a system that offers cost optimization for energy use in Nigeria. The solution expected in the residential area of Nigeria is supposed to monitor the use of energy that can be stored in a database to access historical data to create awareness [47]. The primary component used in this paper is the ACS712 current sensor which measures both the alternating current and the direct current. The components used include Arduino UNO, an LCD, a ACS712 current sensor, a $4 \times 4$ matrix calculator, and a GSM module. This system can be monitored on the LCD screen and also on the Cloud Application Consumer web server.

It was designed using the HTML/PHP script. The GSM module can also monitor the data, where the data will be texted to the numbers installed. The system was limited to a small communication range.
TABLE 2: Average current reading on a multimeter without PMAS and on one with a PMAS adapter [46].

\begin{tabular}{lcc}
\hline Appliances & $\begin{array}{c}\text { Current reading from } \\
\text { multimeter (A) }\end{array}$ & $\begin{array}{c}\text { Current reading with } \\
\text { PMAS adapter }\end{array}$ \\
\hline $\begin{array}{l}\text { Air } \\
\text { conditioner }\end{array}$ & 12.5 & 12.9 \\
$\begin{array}{l}\text { Hair dryer } \\
\begin{array}{l}\text { Induction } \\
\text { cooker }\end{array}\end{array}$ & 5.1 & 5.5 \\
Microwave & 3.7 & 3.8 \\
\hline
\end{tabular}

The authors have designed a home control and monitoring system where this system was developed as an embedded micro web server and a remote application by using a smartphone. This project is aimed at reducing costs and saving energy. Other than that, the authors wanted to control the device and the home environments were also being monitored to maintain the amount of desired energy consumption. Arduino UNO with the ESP $8266 \mathrm{WiFi}$ module and the clamp current transformer with relay have been used for the appliances. A different AT command should also be presented by GSM-based communications as well as home control. The disadvantage of the system is that consumers are not given any user interface to display, and users have to remember the AT command to control the connected device. The architecture is shown in Figure 4 [48].

The implementation of the system is divided into two parts that are implemented and combined. It consists of 


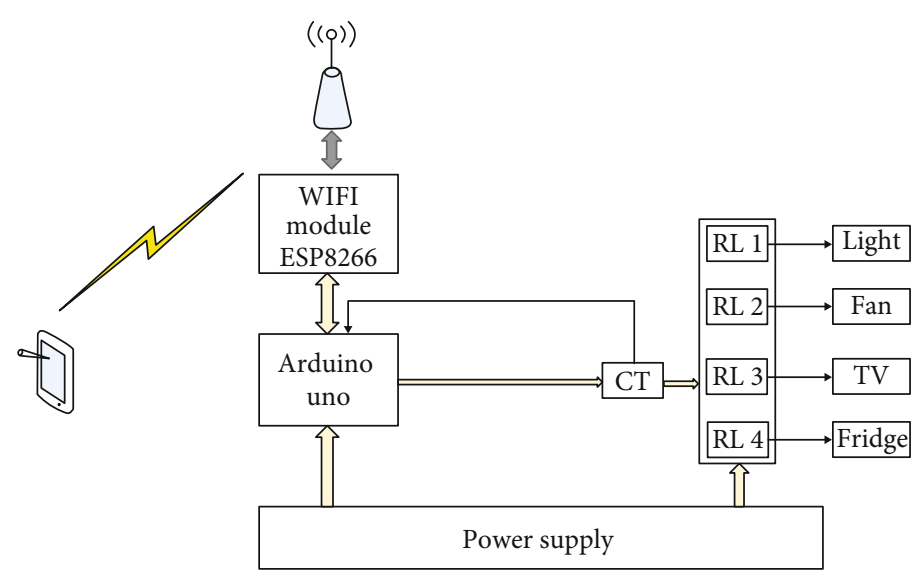

Figure 4: The block diagram of IoT-based control [48].

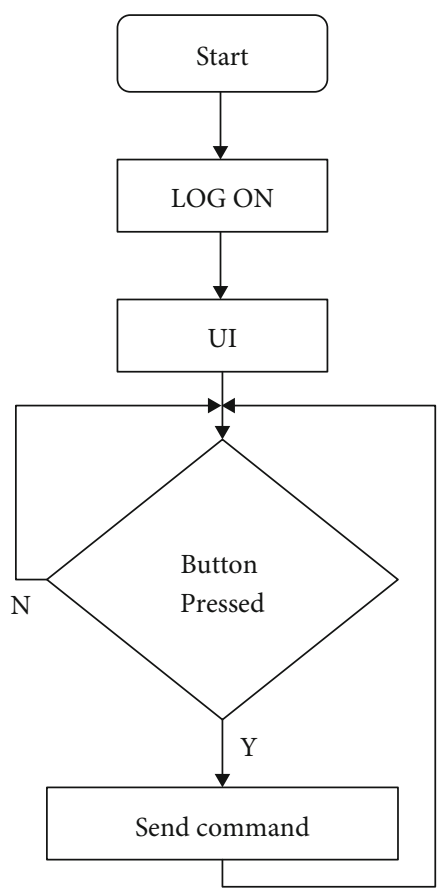

Application

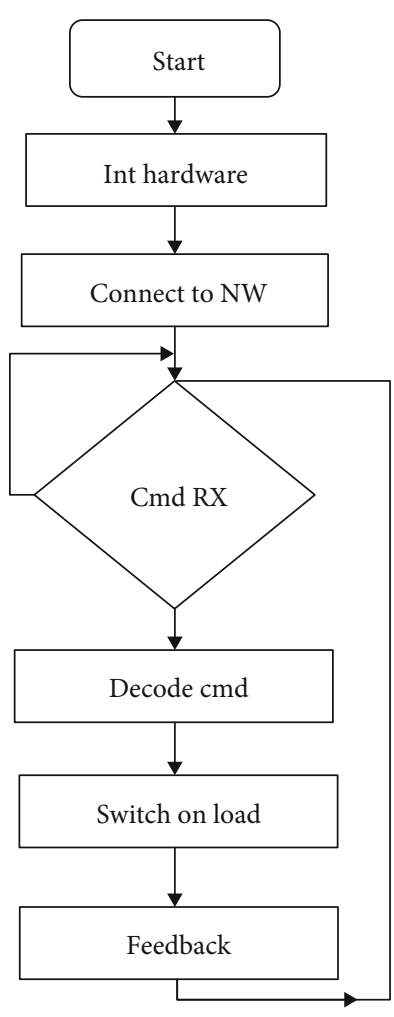

Embedded

FIgURE 5: The flowchart of the IoT-based control.

mobile phones, an android application for the app part, and an Arduino board with a WiFi module in the home gateway for the second embedded part. Figure 5 shows the suggested flow diagram for both systems, respectively.

This study's advantages are that it can reduce human efforts, save power, and low cost. This project's application is a control and monitoring system using different home appliances based on IoT, and this system can be improved further for industrial purposes. It can also be used in offices by using the mobile application [46]. The authors found that a current transformer is not efficient because the reading was not directly from the cable. The impacts of this research in comparison with the proceeding technology in the context of sustainability are summarized below:

(i) Bigger data storage that increases the communication speed by using the WiFi module ESP8266 instead of using ZigBee communication technology

(ii) Targeting IoT sustainable technology that evolves with future upgrading

(iii) Low cost and less complicated technology 


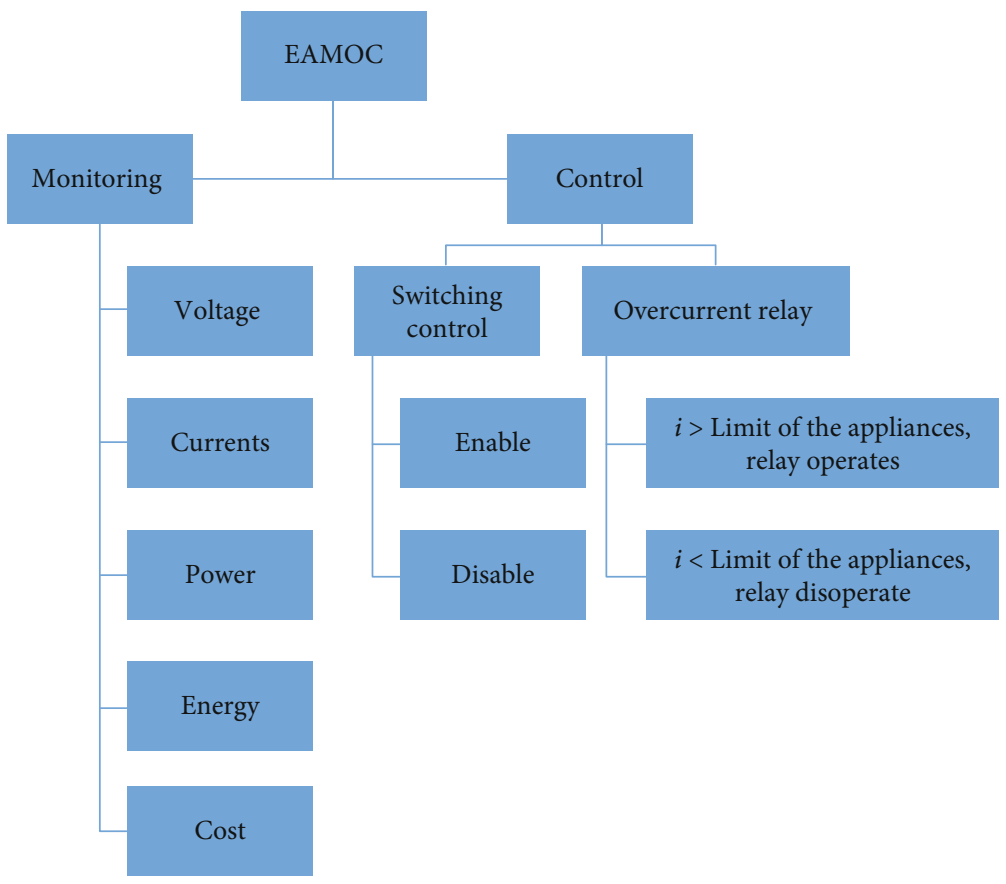

FIgURE 6: Flow diagram of a low-cost Smart HAMCoS based on IoT.

(iv) Real-time usage and fulfilling customer requirements

IoT application to the electrical systems opens the opportunities for short, medium, and long-term adaptation processes, which takes its course as smart technology can be driven from household appliances to the large-scale industry. As seen from the results achieved from this research work, IoT proves real practical application capabilities, leading to sustainable and mature technology.

2.2. Research Gaps. Based on the literature review, the electricity usage monitoring system is aimed at creating awareness for users to be more concerned with energy consumption in the home in general and every appliance specifically. The research was conducted for the monitoring system in which monitoring rules on IoT can be more efficient and manageable. Based on the papers reviewed, there are conflicting results among the authors. The WiFi communication unit used in the monitoring system is the Zigbee transmitter as it is designed to transmit a small amount of data over a short distance from the network. It is considered to be slow as the maximum speed is $250 \mathrm{kbps}$. However, Zigbee has an inoperability problem where it can interfere with one and another. The main research question is what design considerations and techniques need to be focused on controlling the power consumption in residential buildings. More studies are needed to find a better solution that can handle bulk data in the long-distance network and efficiently monitor the energy of house appliances. The existing works using sensors in the system are yet to function efficiently as the sensor in direct contact. As a result, the percentage of error increases in monitoring the current units for the household appliances. A more in-depth study is required to find a better solution to get accurate results.

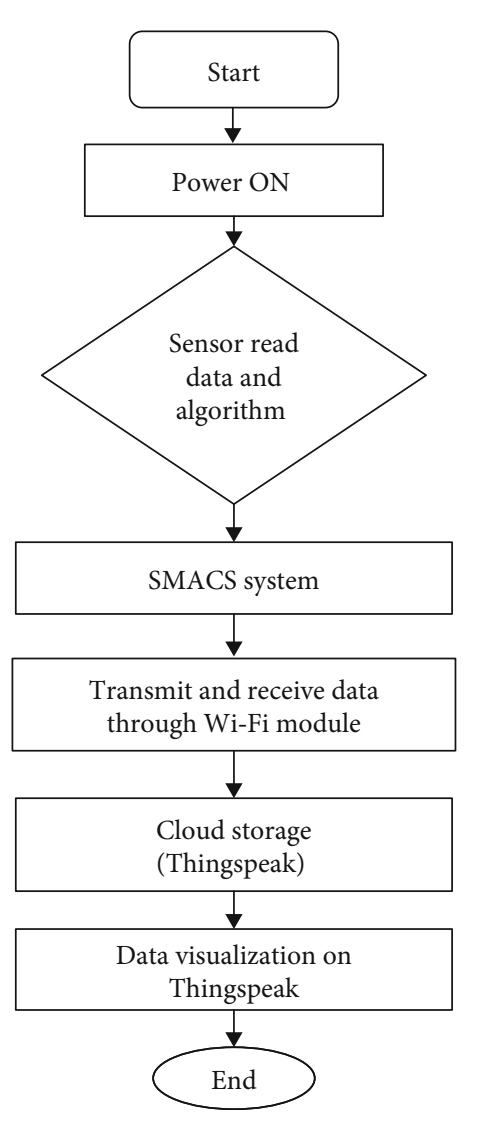

FIgURE 7: The flowchart of SMACS system. 


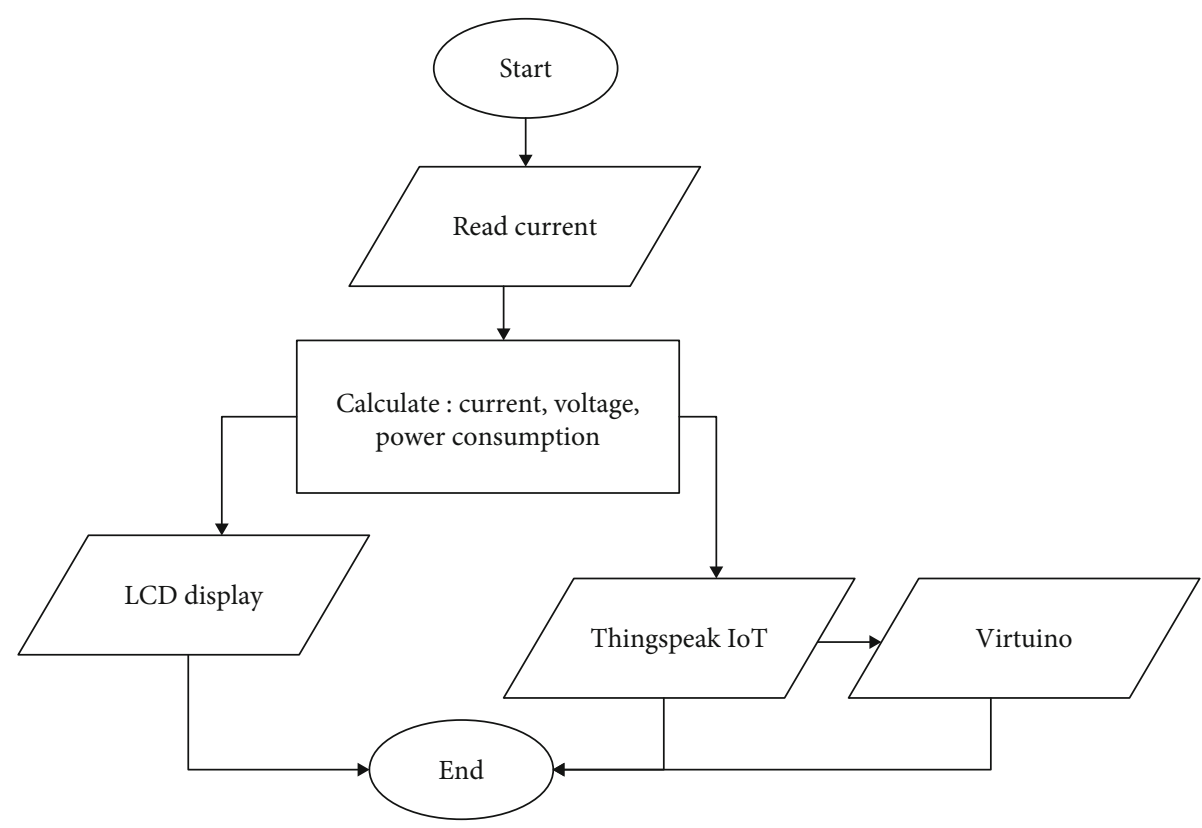

FIGURE 8: Monitoring system of SMACS flowchart.

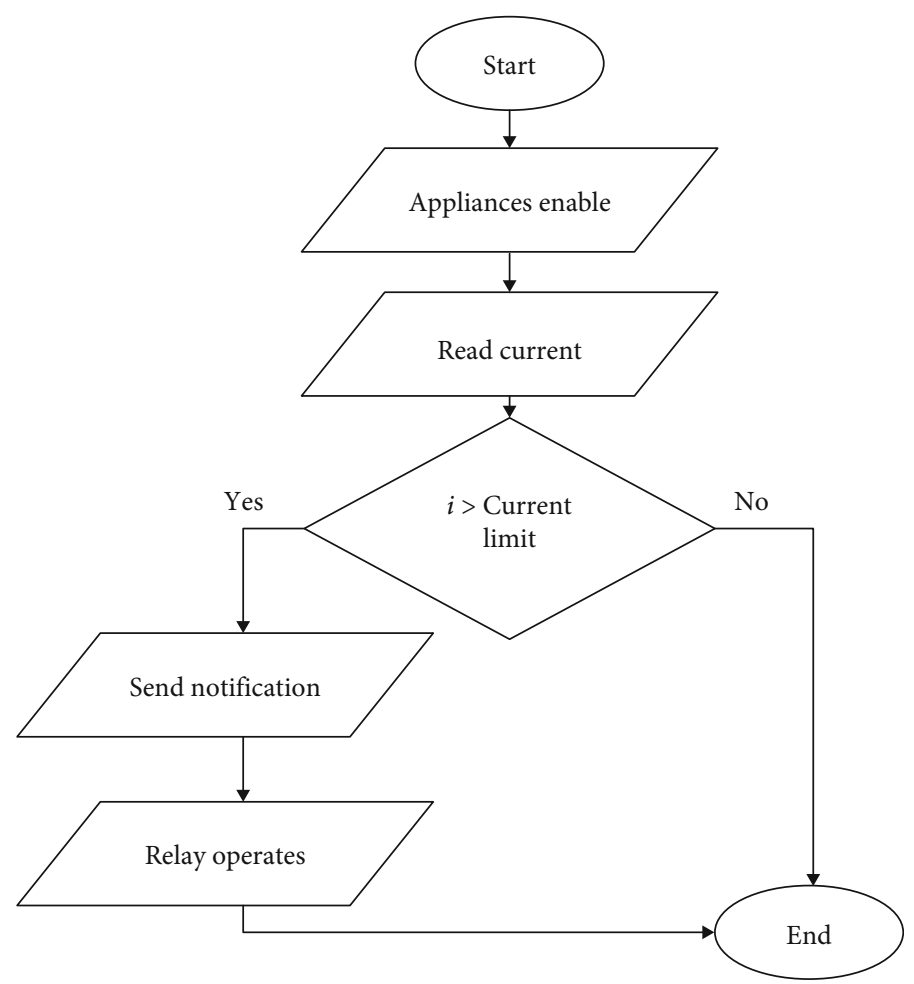

Figure 9: Control system of SMACS.

\section{Materials and Methods}

The developed SMACS in this paper discussed the monitoring system, control system, implementation of IoT, appliances, and basic electrical theory. Figure 6 presents the proposed flow diagram, consisting of five specific parts that are explained in the following discussion.
3.1. Monitoring System. Since the user appliances and the power consumption have increased in residential and commercial sectors, it is imperative to monitor and measure the electric system and appliances. As the focus of this study, the residential sectors are made up of small energy consumers and users, such as houses and apartments [34]. A power monitoring system can help users or consumers to 


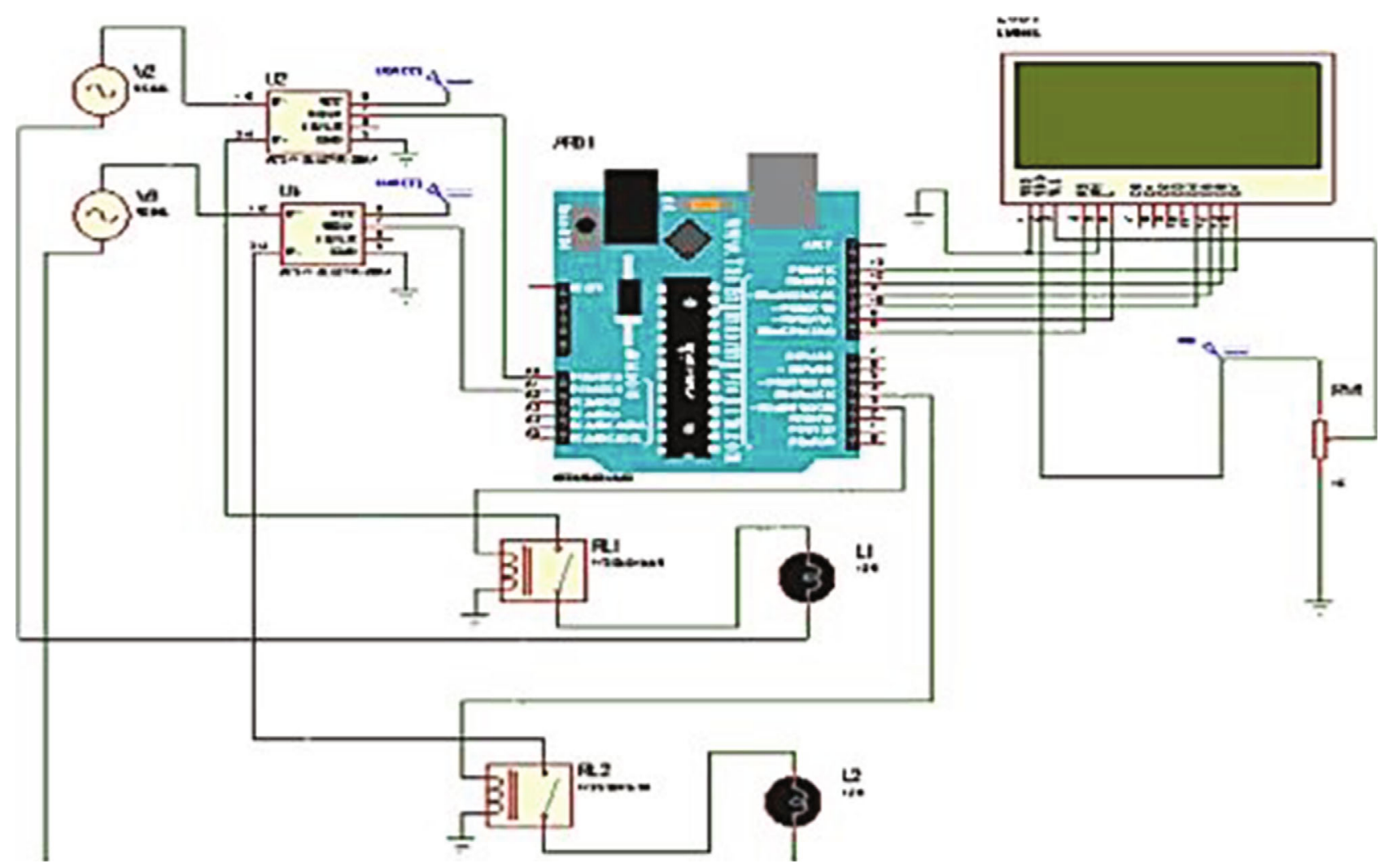

Figure 10: System design of SMACS.

monitor their usage of electricity efficiently. Improvement of electricity efficiency usage can be achieved by reduction due to changing habits [8].

In this modern era, monitoring systems must sort the data and record and exploit them to get the intrinsic information shown in legit and innovative ways and access the Internet to visualize it anywhere [4]. The monitoring system can be considered a user-friendly and unified solution for reliable electricity management [5]. This system can help users to change their usage by providing real-time feedback [6].

3.2. Control System. Electric equipment's operational status control can reduce buildings' power consumption cost and improve the buildings' electrical energy to an effective and reliable ratio. The power control system can help the user manage their electricity more effectively and efficiently as everything can be controlled. This system can give commands directly from the remote control device [5].

3.3. Implementation of IoT. Thing-speak has been used for the development of IoT which is compatible with the ESP $8266 \mathrm{WiFi}$ module. It can also show, analyze, and calculate the required data and store them publicly or privately in the cloud. Thing-speak is used as a medium to analyze and store data from the sensor-based IoT. It can be analyzed remotely via either a laptop or cell phone as long as WiFi is attached. To integrate Thing-speak with Arduino and the ESP8266 WiFi module, some prerequisites need to be done. The most crucial process is the command. Before the

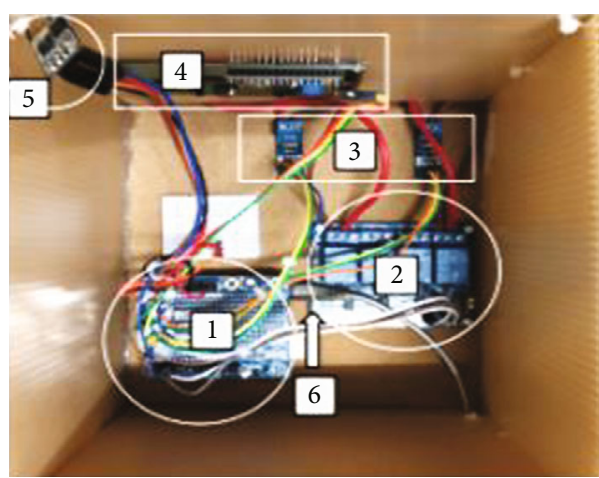

Figure 11: Testbed scenario of SMACS.

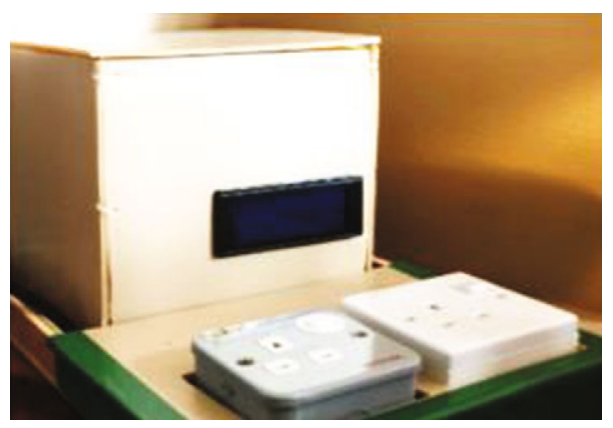

FIGURE 12: Complete SMACS prototype. 
command is done, the Thing-speak account needs to be created. Only authorized persons can access the IoT application through Arduino and the ESP8266 WiFi module using the account created.

\section{Design Consideration}

The first step of this research work is system design. A flowchart is constructed to visualize the project flow, and the process is clearer and more understandable. Figure 7 presents the general system flow of SMACS which shows that, when the system is powered up, the sensors will read the data and all the processes will be executed. The data will be transmitted and received through the WiFi module and then stored on the cloud storage. The data will be visualized in both web servers and mobile applications. As far as SMACS is concerned, it is a system that monitors the current, voltage, and power consumption usage of the appliances that can be monitored on LCD and also based on the Internet of Things by using Thing-speak. The WiFi module ESP8266 is used as a medium to transmit and receive data using WiFi. SMACS for household appliances is divided into 2 parts: one major part for the monitoring system and one minor part for the control system. This system is supplied using $12 \mathrm{Vdc}$ or $240 \mathrm{Vac}$ with an AC to DC converter. The ACS712 current sensor module is used to measure the current, and the value of the current is used to calculate the power consumption. Relay is used in the control system part where it acts as protection, and it cuts off and delays the current when overcurrent occurs. Figures 8 and 9 show the flowchart of the monitoring system and control system, respectively. As shown in Figure 10, the design of SMACS consists of Arduino UNO, a liquid crystal display (LCD), an ACS712 current sensor module, relays, and AC sources. The components can be selected from the library of the software so that the simulation result will be the same as the prototype.

4.1. System Configuration (Thing-Speak). Thing-speak is an Internet of Things open-source application. It stores data and retrieves it via HTTP protocol over the Internet or Local Area Network (LAN) [44]. Thing-speak is compatible with the ESP 8266 WiFi module, and it is also used to show, analyze, and calculate the data and store it publicly or privately in the cloud. In this project, Thing-speak is used to analyze and store data from the sensor based on IoT. It was analyzed through both laptops and mobile phones anywhere and in any place as long as $\mathrm{WiFi}$ is connected. To integrate Thing-speak with Arduino and the ESP8266 WiFi module, some prerequisites need to be done. The most crucial process is the command. Before the command is done, the Thing-speak account was created.

Figure 8 presents the SMACS monitoring system, where it first reads the current status then calculates the current, voltage, and total power consumption. These estimations can be monitored in the LCD and also in the apps using the Thing-speak IoT module. Figure 9 shows the flowchart of the proposed SMACS control system, enabling the appliances to read the current and check its limit. If the expected

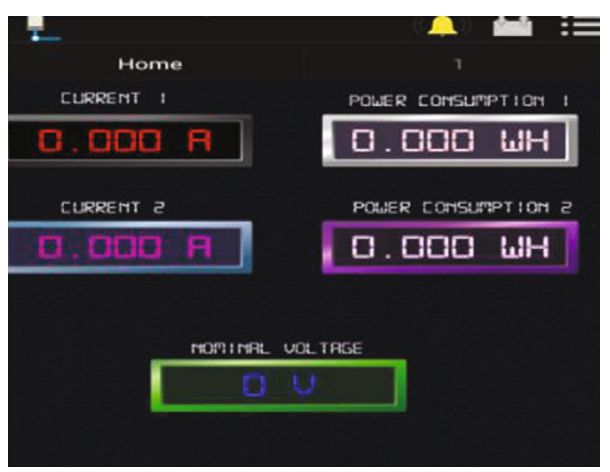

FIgURE 13: Numerical display from Thing-speak.

TABle 3: Component shown in numbering.

\begin{tabular}{lc}
\hline Number & Component \\
\hline 1 & Arduino UNO \\
2 & 5 V relay \\
3 & ACS712 current sensor \\
4 & LCD $20 \times 4$ I2C \\
5 & ESP8266 WiFi module \\
6 & Power adapter \\
\hline
\end{tabular}

TABle 4: Voltage level.

\begin{tabular}{lc}
\hline Voltage level & Supply voltage \\
\hline Low voltage & \\
Single phase & $240 \mathrm{~V}$ \\
Three phases & $415 \mathrm{~V}$ \\
\hline
\end{tabular}

current is greater than the limit, then it sends the notifications to the relay operation.

4.2. Hardware Configuration. The circuitry diagram is shown for the proposed SMACS in Figure 10. After designing and simulating the system using Proteus and Arduino IDE, the next step is to build and fabricate the prototype using the correct configurations. The first fabrication is developed to test and make sure the system is functioning. Figures 11 and 12 show the experimental testbed of SMACS. All the connections are based on the design created on Proteus. Two supply sockets are used as the power source for the appliances, and LCD and Thing-speak are used to display and store data, respectively. As shown in Figure 11, the hardware testbed scenario of SMACS is stated in numbering; the details are shown in Figure 13, and the data is presented in Table 3. This testbed was conducted at the university laboratory with load variations.

4.3. System Parameters. The main system parameters used for the performance evaluation and calculation are discussed here. The residential voltage rating with the configuration is presented in Table 4, where the voltage levels from the low side to the high side for a single phase and for three phases are shown. 
TABLE 5: Comparison between ESP8266 and Zigbee transmitter.

\begin{tabular}{lcc}
\hline ESP8266 WiFi module & Zigbee module & Comparison \\
\hline Wireless local area network (WLAN) area networks & Restricted to wireless personal area networks (WPAN), & Network range \\
with an average range between 30 and 100 meters & reaching $10-30$ meters in usual applications & Operating frequencies \\
$2.4 \mathrm{GHz}, 5 \mathrm{GHz}$ & $900-928 \mathrm{MHz}, 2.4 \mathrm{GHz}$ & Data rate \\
Up to $54 \mathrm{mbps}$ & $250 \mathrm{kbps}$ low data rate & . \\
\hline
\end{tabular}

TABLE 6: Current comparison and percentage error of proposed SMACS.

\begin{tabular}{lccc}
\hline Appliances & Actual current (A) & $\begin{array}{c}\text { Current reading from } \\
\text { Thing-speak (A) }\end{array}$ & $\begin{array}{c}\text { Percentage error } \\
\text { of current (\%) }\end{array}$ \\
\hline Hair dryer & 2.461 & 2.476 & 0.60 \\
Dry iron & 4.175 & 4.190 & 0.41 \\
Induction cooker & 3.905 & 3.890 & 0.38 \\
Water heater & 6.78 & 6.80 & 0.29 \\
\hline
\end{tabular}

As shown in Table 4, a low voltage side is selected for this system where a single-phase housing of the scope of SMACS is a $240 \mathrm{Vac}$ from the power supply. The crucial part of the household appliances is not on the voltage side but on the current side. Current is an electrical charge that carries flow. A simple way of defining this is that current is a charge over time. Current can be categorized into 2 parts: direct current (DC) and alternating current (AC). DC flows only in one direction, whereas AC periodically changes in direction. The root mean square (RMS) current is used in the calculation since AC is constantly changing its value. The RMS current is calculated as follows:

$$
I_{\text {r.m.s. }}=\frac{I_{\max }}{\sqrt{2}},
$$

where $I_{\text {r.m.s. }}$ is the root mean square of the current in A, $I_{\max }$ is the maximum value of current in $\mathrm{A}$.

Other than that, the other important parameter in SMACS is power consumption. Power is an electrical energy flow for the unit time. It can also be defined as the multiplication of voltage, current, and power factors. The formula for power is shown in equation (2) and equation (3):

$$
\begin{gathered}
P=V I \cos \theta, \\
Z=\frac{V}{I} \cos \theta,
\end{gathered}
$$

where $P=$ power in $\mathrm{W}, I=$ current in $\mathrm{A}, \cos \theta=$ power factor , $Z$ is the impedance of the system.

Voltage and power factors are assumed in SMACS, where it is fixed to $240 \mathrm{Vac}$ and $90 \%$, respectively. It is the nominal value for residential areas, where all the values are based on active power.

\section{Result and Discussions}

Most researchers have been using the Zigbee transmitter as a medium to transmit and receive data and cloud storage in the current work. Smart monitoring and control system (SMACS) for household appliances have chosen the ESP8266 WiFi module as a WiFi connection medium. ESP8266 has shown a great work of transmitting and receiving data in this study compared to the Zigbee transmitter. The comparison between the Zigbee transmitter and the WiFi module is shown in Table 5.

Table 6 shows that the ESP8266 WiFi module can facilitate higher data rates at $54 \mathrm{Mbps}$, and the operating frequencies are $2.4 \mathrm{GHz}$ and $5 \mathrm{GHz}$. Whereas, the Zigbee operates in $900-928 \mathrm{Mhz}$ and also $2.4 \mathrm{GHz}$, and the data rate is $250 \mathrm{kbps}$. Therefore, it can be summarized that ESP8266 is better than Zigbee in terms of range, data rate, and operating frequencies. It is also very efficient and accurate in current sensing, as the current sensor is the most crucial in this module.

5.1. Accessing ESP8266 WiFi Module with Thing-Speak in Web Server. SMACS was tested with the Thing-speak using the $\mathrm{WiFi}$ module to transmit and receive the data from the sensors and Arduino UNO. The first step was to sign up for the Thing-speak account to have ownership of the system. Then, the channel is created to know the amount of data to be monitored. As in this system, four data were visualized and listed as follows: field 1-current 1; field 2 -power consumption 1; field 3-current 2; field 4-power consumption 2; and field 5-nominal voltage. It is shown in Figures 14 and 15 that the channel status is either in the form of a chart and numerical display. Figure 14 displays the chart from Thing-speak.

5.2. Mobile Application. Virtuino mobile application was made to monitor the usage wirelessly and remotely. The mobile application that was used in this is Virtuino. It is an application that can be used as a monitoring system for SMACS. Virtuino can be linked with the Thing-speak web server. It reads the real-time data from Thing-speak and visualizes it on the mobile application; however, Virtuino cannot visualize historical data itself. The data can be read on the mobile application as long as the mobile or $\mathrm{WiFi}$ 


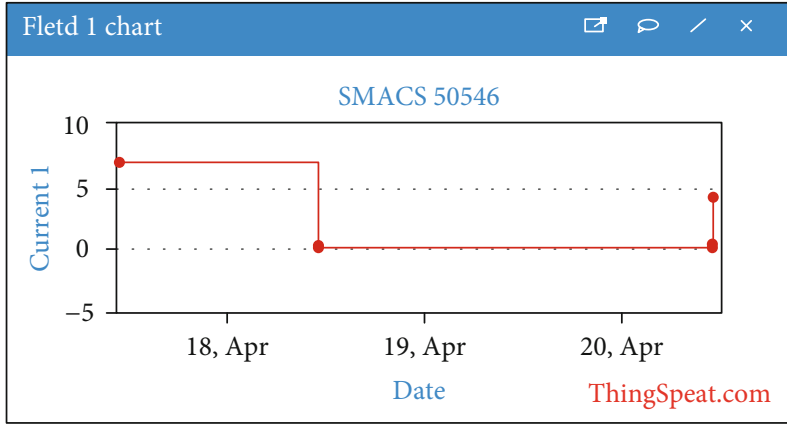

Figure 14: Chart display from Thing-speak.

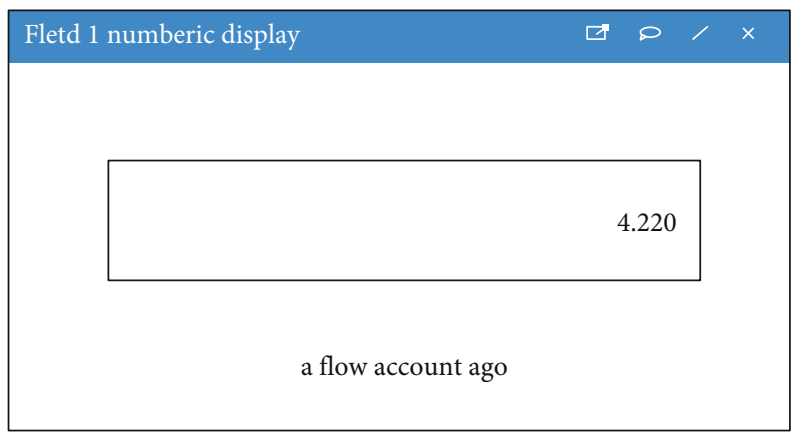

FIgURE 15: Complete visualization of SMACS in Virtuino.

network connection is fast. Historical data can only be accessed on the Thing-speak web server. Figure 16 showed the completed visualization of Virtuino on mobile phones. On the other hand, Virtuino can trigger an alarm whenever an overcurrent occurs. The alarm will produce a sound to trigger awareness to the users about the overcurrent. The value set in this system was $12 \mathrm{~A}$, where it triggered the alarm before the limit of the current value reaches $13 \mathrm{~A}$. This is done to create awareness and avoid any damage or danger to either the appliances or the users themselves. When the value surpasses $13 \mathrm{~A}$, the relay will operate, where the switch will be disabled for a few seconds.

5.3. Data Comparison with Different Household Appliances. A comparison between different types of household appliances were accomplished to identify the accuracy of the proposed system. A few appliances were selected with varying values of current. Table 7 shows the appliances that have been chosen for system testing.

The higher the current value, the more accurate the result of SMACS is presented in Figures 16-23. The percentage of error for the bulb is $2.5 \%$, where the current is the smallest among the appliances chosen. In comparison to the highest value of current among the appliances chosen, which is the water heater, the percentage error is only $0.15 \%$. The value is approaching zero, which is considered to be accurate. This proposed system is considered to be efficient and successful. The data gathered from the monitoring system were tested for 5 minutes for every 30 seconds. The percentage error is calculated.

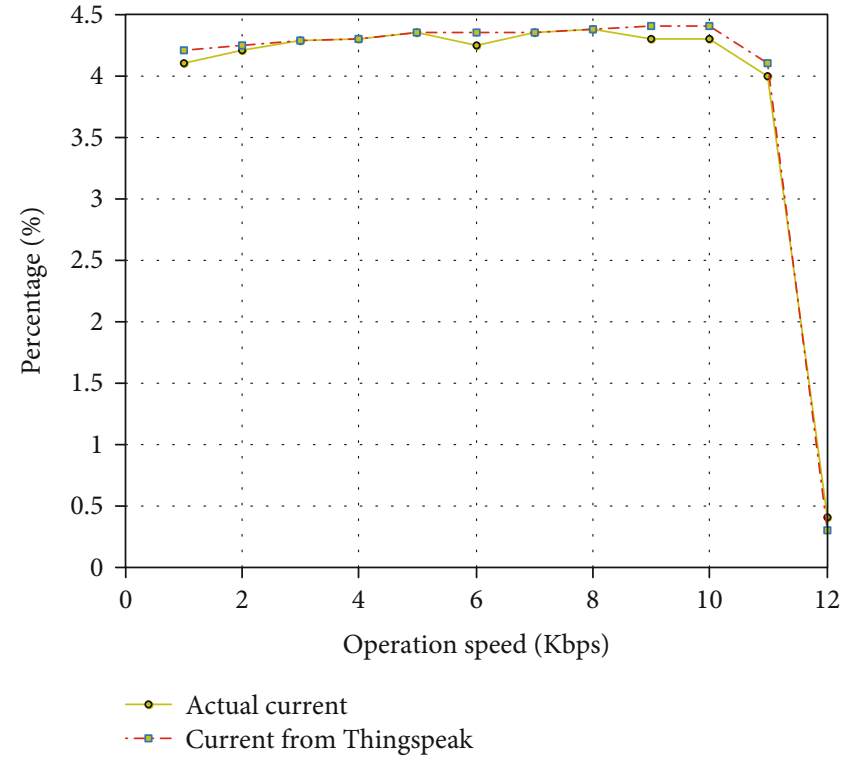

FIGURE 16: Current percentage error of SMACS.

TABLE 7: Household appliances used for testing.

\begin{tabular}{lc}
\hline Appliances & Power rating \\
\hline Bulb & $100 \mathrm{~W}$ \\
Hair dryer & $900 \mathrm{~W}$ \\
Dry iron & $1000 \mathrm{~W}$ \\
Rice cooker & $1000 \mathrm{~W}$ \\
Water heater & $1500 \mathrm{~W}$ \\
\hline
\end{tabular}

As presented in Figure 21, the higher the value of the current, the more accurate the result will be using SMACS. The percentage of error for the bulb is $2.5 \%$, where the current is the smallest among the appliances chosen.

According to the paper on microcontroller-based power monitoring and switching device (PMAS) for appliances over a ZigBee network [46], the system called PMAS as shown in Figures 22 and 23 show the percentage error of each system. It can be seen that the performance of the proposed SMACS is preferably accurate and has very low error compared to the existing PMAS. The detailed comparison is presented in Table 8, where it can be seen that the existing PMAS has chosen a few appliances quite similar to the proposed system. The data can be analyzed to show that PMAS is considered successful, but according to Table 7, the percentage error of currents is relatively large compared to SMACS. Table 6 shows the average value of current and its percentage error of SMACS.

5.4. Discussion and Limitation of This Work. The results obtained are based on a constructed circuit that is simulated using real-life power system data and hardware prototype trials at the power socket. Monitoring power consumption problems are pervasive in household electric appliances. Therefore, a sustainable design and implementation of smart 


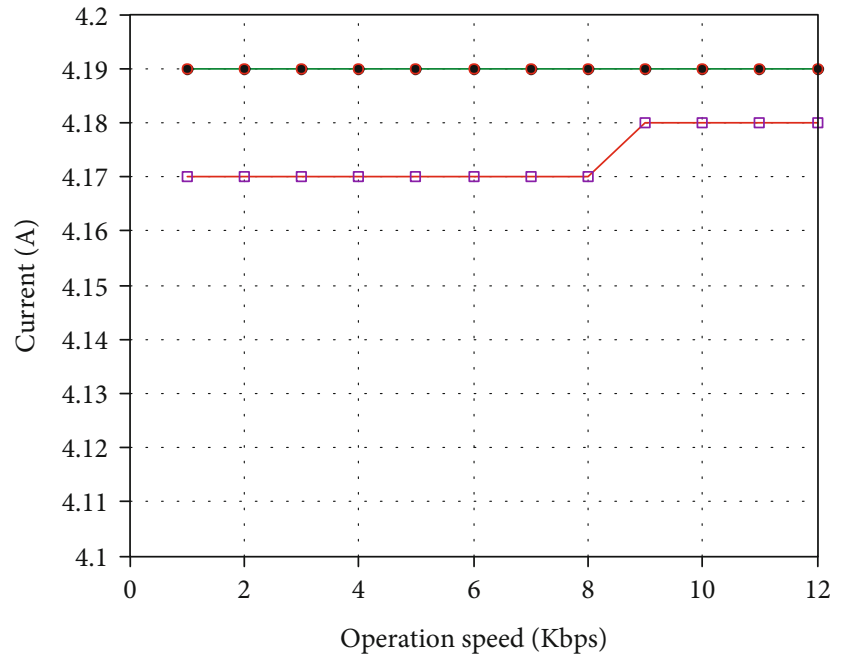

$\rightarrow$ Actual current

$\square$ Current from Thingspeak

FIgURE 17: Current test comparison for rice cooker appliance.

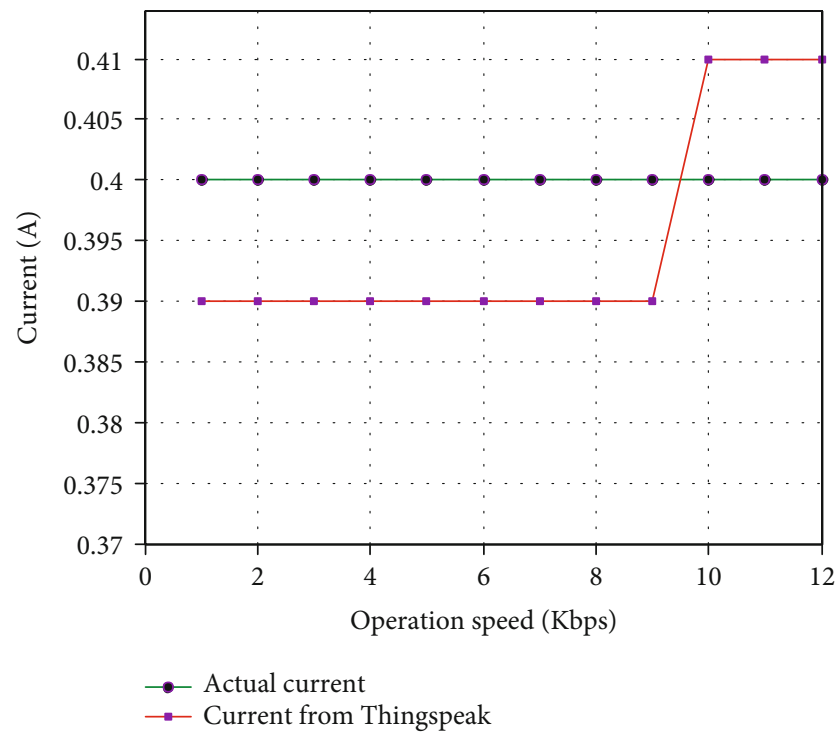

Figure 18: Current test comparison for water heater appliance.

electricity management of every consumption have been top prioritized to reduce usage and cost for a sustainable society $[9,11]$. The proposed smart home electricity monitoring system is sustainable that utilizes the most advanced Internet technologies to impact electric consumption monitoring performance. Monitoring the consumption of electrical appliances, including advanced digital appliances, can directly regulate the usage of the air conditioner, water heaters, heaters, dry iron, washing machine, music systems, and lighting systems. Therefore, the proposed system can positively improve lifestyles and habits for a sustainable urban lifestyle with the accessible functionalities that can connect the house to an extraordinary remote monitoring center or to a mobile device through a mobile app; it is environmentally friendly. Using the proposed system can create

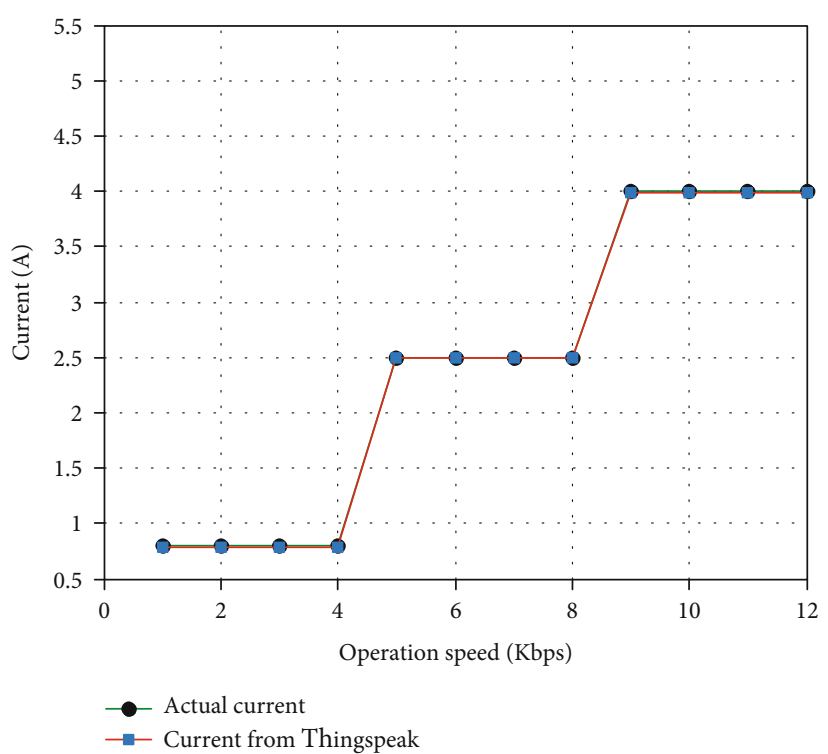

FIgURE 19: Current test comparison for $100 \mathrm{~W}$ lighting appliance.

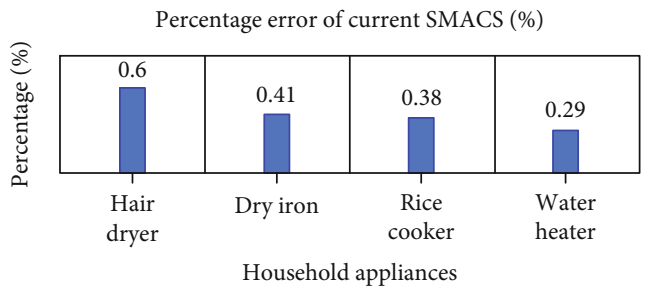

FIGURE 20: Current test comparison for hairdryer appliance.

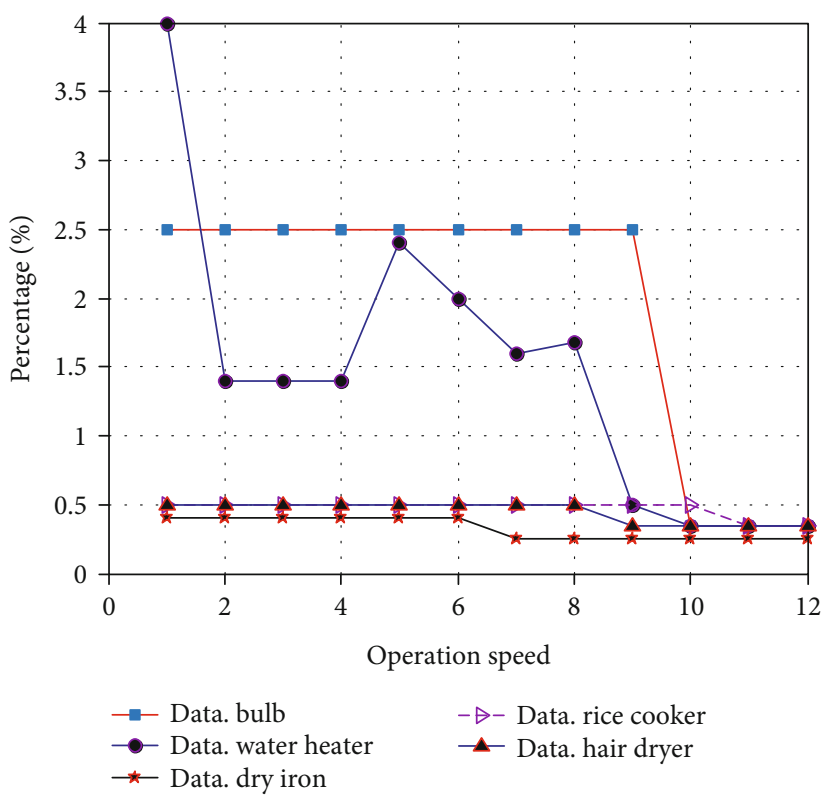

FIgURe 21: Percentage error comparison with different appliances. 


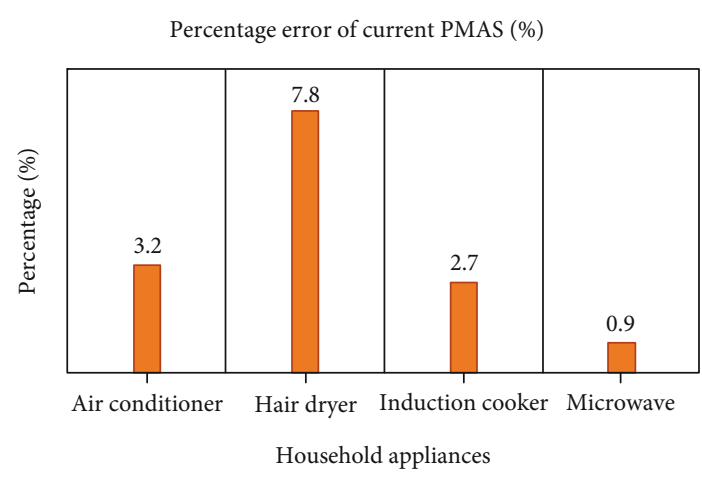

Figure 22: Percentage error of PMAS.

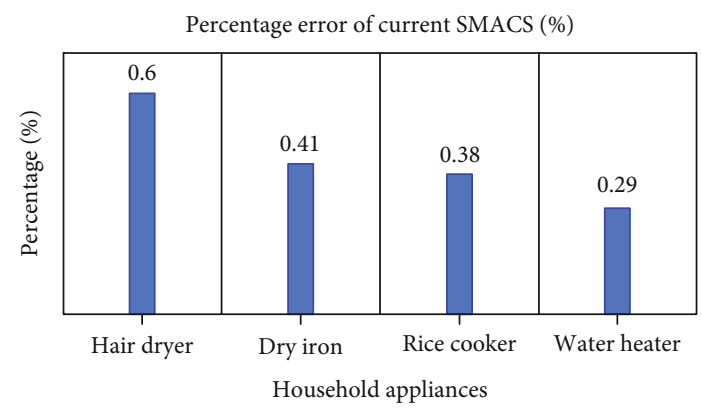

Figure 23: Percentage error of SMACS.

TABLE 8: Current comparison and percentage error of PMAS [44].

\begin{tabular}{lccc}
\hline Appliances & $\begin{array}{c}\text { Current } \\
\text { reading from } \\
\text { multimeter (A) }\end{array}$ & $\begin{array}{c}\text { Current reading } \\
\text { with PMAS } \\
\text { adapter (A) }\end{array}$ & $\begin{array}{c}\text { Percentage } \\
\text { error of } \\
\text { current (\%) }\end{array}$ \\
\hline $\begin{array}{l}\text { Air conditioner } \\
\text { Hair dryer }\end{array}$ & 12.5 & 12.9 & 3.2 \\
$\begin{array}{l}\text { Induction } \\
\text { cooker }\end{array}$ & 5.1 & 5.5 & 7.8 \\
Microwave & 3.7 & 3.8 & 2.7 \\
\hline
\end{tabular}

awareness of energy usage for the appliances like air conditioners, bulbs, and other home appliances. The significant advantage is that the proposed system can be customized at the controller for future expansion. However, IoT-based systems for the smart home have a few disadvantages, especially with regard to the challenge of privacy, bandwidth, and Internet security.

Some limitations for this work, such as the voltage, were assumed to be $240 \mathrm{Vac}$ since no sensor was included for the voltage sensor. However, in our future study, a voltage sensor is considered to measure the real-time voltage from the incoming voltage supply. Thus, the accuracy of the result can be improved further. Other than that, undervoltage and overvoltage detection can also be included if the voltage sensor is used. The control system can be improved further by considering time scheduling, remote switching, automatic switching, temperature switching, and sensor controlling. This will help the users to reduce electricity consumption as well as to make life easier.

\section{Conclusions}

This paper has proposed and developed an IoT-based smart monitoring and control system for household appliances. The methodology and processes are designed and created to provide a useful and crucial solution in a monitoring and control system. The proposed system performed better in measuring and monitoring the current, voltage, and power consumption. This is because the proposed SMACS considered the system process design with the hardware selection of the ESP8266 WiFi module, ACS712 current sensors, and $5 \mathrm{~V}$ relays. The entire communication has been done through the WiFi module to display the data on web servers, Thing-speak, and mobile applications like Virtuino. The data are also monitored in real time and historically, where the data were stored in the cloud database. The monitoring system makes the system more accessible for customers to view consumption rates clearly in real time. The control system of SMACS can fulfill the requirement of safety, can monitor appliances in the household individually or collectively, and can create awareness on electricity consumption. The voltage and current will be monitored in a wider scale in the smart house environment in future work.

\section{Data Availability}

All data has been supplied with the manuscript.

\section{Conflicts of Interest}

The authors declare no conflict of interest.

\section{Acknowledgments}

This paper was funded by Universiti Kebangsaan Malaysia (UKM) under grant nos. FRGS/1/2020/ICT03/UKM/02/6 and GGPM 2020-028.

\section{References}

[1] J. S. Hassan, R. M. Zin, M. Z. A. Majid, S. Balubaid, and M. R. Hainin, "Building energy consumption in Malaysia: an overview," Jurnal Teknologi, vol. 70, no. 7, pp. 33-38, 2014.

[2] S. Engineering and D. Systems, "Monitoring and reducing the consumption of home electric appliances December 2013," Monitoring and reducing the consumption of home electric appliances, p. 53, 2013.

[3] E. Effah, F. L. Aryeh, W. K. Kehinde, and E. Effah, "GSMBased Home Appliances Control System for Domestic Power Users in Ghana," Ghana Journal of Technology, vol. 1, no. 1, pp. 25-30, 2016.

[4] T. Teng-Fa and K. Cheng-Chien, "A smart monitoring and control system for the household electric power usage," in 2013 IEEE PES Asia-Pacific Power and Energy Engineering Conference (APPEEC), Hong Kong, China, 2013.

[5] Y. R. Tan, F. W. Akashah, and N. Mahyuddin, "The analysis of fire losses and characteristics of residential fires based on investigation data in Selangor, 2012-2014," in MATEC Web of Conferences, vol. 66, p. 00109, 2016. 
[6] S. S. van Dam, C. A. Bakker, and J. D. M. van Hal, "Home energy monitors: impact over the medium-term," Building Research \& Information, vol. 38, no. 5, pp. 458-469, 2010.

[7] W. A. Jabbar, T. K. Kian, R. M. Ramli et al., "Design and Fabrication of Smart Home With Internet of Things Enabled Automation System," IEEE Access, vol. 7, pp. 144059144074, 2019.

[8] K. Gram-Hanssen, "Efficient technologies or user behaviour, which is the more important when reducing households' energy consumption?," Energy Efficiency, vol. 6, no. 3, pp. 447-457, 2013.

[9] A. G. Paetz, E. Dütschke, and W. Fichtner, "Smart homes as a means to sustainable energy consumption: a study of consumer perceptions," Journal of Consumer Policy, vol. 35, no. 1, pp. 23-41, 2012.

[10] J. Walzberg, T. Dandres, N. Merveille, M. Cheriet, and R. Samson, "Assessing behavioural change with agent-based life cycle assessment: application to smart homes," Renewable and Sustainable Energy Reviews, vol. 111, pp. 365-376, 2019.

[11] J. M. Batalla, A. Vasilakos, and M. Gajewski, "Secure Smart Homes," ACM Computing Surveys, vol. 50, no. 5, pp. 1-32, 2017.

[12] E. Carroll, E. Hatton, and M. Brown, Residential Energy Use Behavior Change Pilot, CMFS project code B 21383, 2009.

[13] M. Trejo-Perea, G. J. R. Moreno, A. Castañeda-Miranda, D. Vargas-Vázquez, R. V. Carrillo-Serrano, and G. HerreraRuiz, "Development of a Real Time Energy Monitoring Platform User-Friendly for Buildings," Procedia Technology, vol. 7, pp. 238-247, 2013.

[14] U. G. Scholar, "Smart Power Flow Monitoring and Control," International Journal of Science Technology \& Engineering, vol. 2, no. 10, pp. 1159-1165, 2016.

[15] E. Mohamed and M. Ibrahim, Smart Energy Monitor, Infinity Tech of Embedded Solution, Mansoura, 2018.

[16] M. J. Watts and S. P. Worner, "Comparing ensemble and cascaded neural networks that combine biotic and abiotic variables to predict insect species distribution," Ecological Informatics, vol. 3, no. 6, pp. 354-366, 2008.

[17] M. Shafiq, Z. Tian, A. K. Bashir, X. Du, and M. Guizani, "CorrAUC: a malicious bot-IoT traffic detection method in IoT network using machine learning techniques," IEEE Internet of Things Journal, vol. 8, no. 5, pp. 3242-3254, 2021.

[18] A. H. Bagdadee, L. Zhang, and M. S. Remus, "A brief review of the IoT-based energy management system in the smart industry," in Artificial Intelligence and Evolutionary Computations in Engineering Systems, pp. 443-459, Springer, 2020.

[19] Z. Ibrahim and R. Hassan, "The implementation of Internet of Things using Test Bed in the UKMNET environment," Asia Pacific Journal of Information Technology and Multimedia, vol. 8, no. 2, pp. 1-17, 2019.

[20] Q.-V. Pham, M. Liyanage, N. Deepa et al., “Deep Learning for Intelligent Demand Response and Smart Grids: A Comprehensive Survey," 2021, http://arxiv.org/abs/2101.08013.

[21] N. Sakib, E. Hossain, and S. I. Ahamed, “A qualitative study on the United States internet of energy: a step towards computational sustainability," IEEE Access, vol. 8, pp. 69003-69037, 2020.

[22] Z. E. Ahmed, M. K. Hasan, R. A. Saeed et al., "Optimizing energy consumption for cloud Internet of Things," Frontiers of Physics, vol. 8, 2020.
[23] R. B. Gubbi and S. Marusic, "Internet of Things (IoT): a vision, architectural elements, and future directions," Future generation computer systems, vol. 29, no. 7, pp. 1645-1660, 2013.

[24] M. B. Rivera, E. Eriksson, and J. Wangel, "ICT practices in smart sustainable cities-in the intersection of technological solutions and practices of everyday life," in EnviroInfo and ICT for Sustainability 2015, Atlantis Press, 2015.

[25] B. Vanderborght, "Technology is not neutral (from the Editor's desk)," IEEE Robotics \& Automation Magazine, vol. 25, no. 1, pp. 4-4, 2018.

[26] Y. Strengers, "Smart energy in everyday life: are you designing for resource man?," Interactions, vol. 21, no. 4, pp. 24-31, 2014.

[27] M. K. Hasan, A. F. Ismail, S. Islam, W. Hashim, M. M. Ahmed, and I. Memon, "A novel HGBBDSA-CTI approach for subcarrier allocation in heterogeneous network," Telecommunication Systems, vol. 70, no. 2, pp. 245-262, 2019.

[28] S. Islam, A. H. Abdalla, and M. Kamrul Hasan, "Novel multihoming-based flow mobility scheme for proxy NEMO environment: a numerical approach to analyse handoff performance," ScienceAsia, vol. 43S, no. 1, pp. 27-34, 2017.

[29] R. A. Mokhtar, R. A. Saeed, H. Alhumyani, M. Khayyat, and S. Abdel-Khalek, "Cluster mechanism for sensing data report using robust collaborative distributed spectrum sensing," Cluster Computing, vol. 4, pp. 1-6, 2021.

[30] M. K. Hasan, M. M. Ahmed, S. S. Musa et al., "An improved dynamic thermal current rating model for PMU-based wide area measurement framework for reliability analysis utilizing sensor cloud system," IEEE Access, vol. 9, pp. 14446-14458, 2021.

[31] B. L. Risteska Stojkoska and K. V. Trivodaliev, "A review of Internet of Things for smart home: challenges and solutions," Journal of Cleaner Production, vol. 140, pp. 1454-1464, 2017.

[32] T. Kılıç and E. Bayır, "An investigation on Internet of Things technology (IoT) in smart houses," International Journal of Engineering Research and Development, vol. 9, no. 3, pp. 196-207, 2017.

[33] H. Ghayvat, S. Mukhopadhyay, J. Liu, A. Babu, E. Elahi, and X. Gui, "Internet of Things for smart homes and buildings: opportunities and challenges," Australian Journal of Telecommunications and the Digital Economy, vol. 3, no. 4, p. 33, 2015.

[34] S. A. Hashmi, C. F. Ali, and S. Zafar, "Internet of things and cloud computing-based energy management system for demand side management in smart grid," International Journal of Energy Research, vol. 45, no. 1, pp. 1007-1022, 2021.

[35] S. Wang, X. Mao, and H. Khodaei, "A multi-objective home energy management system based on Internet of Things and optimization algorithms," Journal of Building Engineering, vol. 33, article 101603, 2021.

[36] M. K. Hasan, S. Islam, M. Shafiq et al., "Communication delay modeling for wide area measurement system in smart grid Internet of Things networks," Wireless Communications and Mobile Computing., vol. 2021, pp. 1-10, 2021.

[37] S. Sharad, P. Bagavathi Sivakumar, and V. Anantha Narayanan, "A novel IoT-based energy management system for large scale data centers," in Proceedings of the 2015 ACM Sixth International Conference on Future Energy Systems, New York, 2015.

[38] Z. S. Ageed, S. R. Zeebaree, M. A. Sadeeq et al., "A state of art survey for intelligent energy monitoring systems," Asian Journal of Research in Computer Science., vol. 27, pp. 46-61, 2021. 
[39] D. P. Rani, D. Suresh, P. R. Kapula, C. M. Akram, N. Hemalatha, and P. K. Soni, "IoT based smart solar energy monitoring systems," in Materials Today: Proceedings, Elsevier, 2021.

[40] M. K. Hasan, M. M. Ahmed, A. H. Hashim, A. Razzaque, S. Islam, and B. Pandey, "A novel artificial intelligence based timing synchronization scheme for smart grid applications," Wireless Personal Communications, vol. 23, pp. 1-8, 2020.

[41] M. K. Hasan, S. H. Yousoff, M. M. Ahmed, A. H. A. Hashim, A. F. Ismail, and S. Islam, "Phase offset analysis of asymmetric communications infrastructure in smart grid," Elektronika ir Elektrotechnika, vol. 25, no. 2, pp. 67-71, 2019.

[42] A. Ponniran, E. Sulaiman, S. A. Jumaat, M. Ishak, M. A. Chulan, and S. Saiman, "A study on electric energy usage at the residential area," In Engineering Conference, pp. 27-28, 2007.

[43] V. Tadavarthy and A. Broota, "Smart power monitoring \& analysis," International Journal of Science and Research, vol. 5, no. 7, pp. 1627-1630, 2016.

[44] P. R. Joshi and M. S. Prof, "IoT based smart power management system using WSN," International Research Journal of Engineering and Technology, vol. 4, no. 6, pp. 783-786, 2017.

[45] M. G. E. Ante, K. A. N. Garcia, B. A. A. Gonzales, J. T. D. Mendoza, and A. C. Roque, "Microcontroller-based power monitoring and switching device for appliances over a ZigBee network," Journal of Telecommunication, Electronic and Computer Engineering, vol. 10, no. 1, pp. 93-97, 2018.

[46] K. C. Okafor, G. C. Ononiwu, U. Precious, and A. C. Godis, "Development of Arduino based IoT metering system for ondemand energy monitoring," International Journal of Mechatronics, Electrical and Computer Technology, vol. 7, pp. 32083224, 2017.

[47] P. Dasan, A. Prabhu, S. Sundaram, and S. Rajan, "A ubiquitous home control and monitoring system using android based smart phone," International Journal Of Computer Science And Mobile Computing, vol. 2, no. 12, pp. 188-197, 2013.

[48] M. Thomas, 13 examples of IoT in environmental sustainability you should know, 2019, https://builtin.com/internet-things/ iot-environment-sustainability-green-examples. 